\title{
MiniCog: A method for administering psychological tests and experiments on a handheld personal digital assistant
}

\author{
J. M. SHEPHARD \\ Harvard University, Cambridge, Massachusetts \\ S. KHO and J. CHEN \\ Bay Area Software, San Francisco, California \\ and \\ S. M. KOSSLYN \\ Harvard University, Cambridge, Massachusetts
}

\begin{abstract}
Because of their minimal cost, size, and weight, handheld personal digital assistants (PDAs) are appealing as a means for administering response time tasks "in the field" or to participants in longitudinal studies who need repeated access to the testing equipment. We have developed a software package that allows investigators to author simple scripts on their desktop computers and administer the compiled tasks on PDAs. MiniCog presents instructions, practice trials with auditory feedback, and visual stimuli in random or fixed sequential orders with fixed or variable durations and intertrial intervals and records single-button responses and response times. Data from each trial are stored with a user ID, user-entered demographics and notes, and a time-and-date stamp, for later upload to a desktop computer. MiniCog performs automatic calculations of mean response time and error rate, available to users with a password and stored with the other data for upload.
\end{abstract}

This article introduces a new software application, MiniCog, that runs on Palm OS and Windows Mobile handheld personal digital assistants (PDAs) and is capable of administering and scoring simple psychological tasks utilizing visual stimuli. Although a number of applications exist for the programming of psychological experiments to be administered on desktop and laptop computers - for example, Presentation (Neurobehavioral Systems, Inc.), E-Prime (Psychology Software Tools, Inc.), PsyScope (Cohen, MacWhinney, Flatt, \& Provost, 1993), and SuperLab (Cedrus Corporation) - and there are a number of PDA applications for administering surveys, making medical diagnoses, keeping medical diaries, and even administering preprogrammed cognitive tests (see, e.g.,

MiniCog, MiniCogWriter, and pdb2csv were designed by J.M.S. and S.M.K. and were programmed by S.K. and J.C. Their development was supported by the National Space Biomedical Research Institute through NASA NCC 9-58. MiniCog and MiniCogWriter are protected by copyright and U.S. patent pending (to Harvard University). Harvard University, S.M.K., and J.M.S. have a potential financial interest in commercial applications of this software. Palm, Palm OS, and Palm Tungsten are among the trademarks or registered trademarks owned by or licensed to Palm, Inc. Microsoft, Excel, Windows, and Windows Mobile are either registered trademarks or trademarks of Microsoft Corporation in the United States and/or other countries. All other brand and product names are or may be trademarks of their respective owners and are used to identify their products or services. Correspondence concerning this article should be addressed to J. M. Shephard, Psychology Department, Harvard University, 832 William James Hall, 33 Kirkland Street, Cambridge, MA 02138 (e-mail: jmsheph@fas.harvard.edu).
PDA Resources, selected by the Hong Kong University Libraries, and Access Co.'s Psychology and Psychotherapy Resources, Lautzenhiser, 2007), there is currently no PDA software available for presenting investigatorprogrammed psychological experiments or tasks relying on visual stimulus presentation and response time (RT) measurements.

The inspiration for this software was the National Space Biomedical Research Institute's need to develop better methods for assessing the cognitive status of astronauts during long-duration spaceflights. An astronaut's job is likely to be very stressful, given the long work hours, the extreme environment, the isolation from friends and family, the demanding task of operating and piloting the spacecraft, the constant close proximity to other crewmembers, and so on. Different types of stress are likely to affect cognitive performance; moreover, the risks associated with cognitive decline on a long-duration mission are severe (an error docking a spacecraft could be fatal).

Our goal was to build a system for administering cognitive assessment tests that would be both inexpensive (so each astronaut could have his or her own) and extremely small and lightweight (space is at a premium in the vehicles in which astronauts travel and work). Desktop computers are expensive and not portable; although laptops are more portable, they are even more expensive than desktop computers. A typical PDA costs under $\$ 500$, is extremely lightweight, fits in a shirt pocket, and can simply be held in one's hand in microgravity, rather than requiring a strap 
to keep it in place. ${ }^{1}$ When data are collected in the field (on Earth), it is much easier and less expensive to carry around three or four PDAs than three or four laptops. One can easily imagine scenarios in which users are loaned PDAs for a number weeks or months for repeated testing, but it is unlikely that an investigator can afford to hand out full-size computers so casually. The portability of PDAs is particularly useful for repeated testing, because users do not have to go anywhere (even home) to get to the computer; they can simply carry it with them at all times. Indeed, the scheduling functions of a PDA are useful in these situations as well.

In addition to portability, we needed to design a system to provide the astronauts with immediate feedback regarding their performance, which requires online data processing. We recognized that feedback would not be required (or even desired) in all cases and, thus, implemented a password to protect access to the results; nevertheless, the summary statistics are stored automatically even if they are not displayed, which can save an investigator time during data analysis. Even some desktop experiment generators do not have this scoring and data-processing capability.

Finally, it was important to design an easy-to-use interface so that even nonprogrammers could quickly implement their own tasks. Just as most desktop experiment generators can be used by investigators who might find programming a task from scratch in C, for instance, rather daunting and time consuming, MiniCog can be used by programming novices to generate quickly and easily RT tasks utilizing visual stimuli.

\section{OVERVIEW}

MiniCog presents instructions, practice trials with auditory feedback (beeps) after incorrect responses, and visual stimuli in random or fixed sequential orders; it allows specification of stimuli durations and intertrial intervals and records single-button responses and RTs. The data from each trial are stored with a user ID, basic user-entered demographics, notes, and self-evaluations of performance, the trial indicator and correct answer, and a time-and-date stamp. MiniCog performs automatic calculations of, among other things, median and mean RTs and RT standard deviations $(S D \mathrm{~s})$ for correctly answered trials, error rates (ERs), and throughput (TP; a measure of efficiency) for each task; it can present these summary statistics to the user after an appropriate password has been entered. These summary statistics, along with the other stored data, can later be uploaded to a desktop computer for more detailed analyses.

The software package consists of a desktop application, MiniCogWriter, that compiles the task scripts and a PDA application, MiniCog, that administers the tasks. The tasks are authored using any commercial off-the-shelf $\mathrm{HTML}^{2}$ editing tool.

\section{SCRIPTING TASKS}

\section{Table Features}

The source files that MiniCogWriter converts to MiniCog tasks are HTML files with certain conventions; we use a table to script the tasks. Figure 1 illustrates a partial script of a variant of a Stroop task (Stroop, 1935), where the PDA users are shown the digits 4, 5, and 6 printed four, five, or six times in a row. There are equal numbers of congruent stimuli (where the digit matches the number of times it is printed; e.g., four 4s) and incongruent stimuli (where the digit does not match; e.g., five 4s). The users' task is to indicate the number of digits in the display, not the name of the digits. The task consists of instructions, followed by practice trials, followed by the test trials (see Figure 1; there is not enough room to display an image of the entire HTML file, and thus the figure contains only a few of the instruction rows).

In the current implementation, the table must have six columns, and the first row must have the headings as shown: Text, Picture, Picture-240×320, Answer, Duration, and Delay. A caption is used to assign a name to the output file. Each row of the table corresponds to one screen on the PDA.

Any text the investigator wants to display must be written in the Text column; a typical PDA screen accommodates approximately 15 lines of text, averaging about 33 characters per line. Text color can be specified using standard HTML, but the size is hardwired to the standard PDA text size; the background is white.

Any images (JPGs, GIFs, or PNGs) that the investigator plans to display, whether stimuli or part of the instructions, are specified in the Picture column (for $320 \times 320$ pixel images for Palms) or the Picture- $240 \times 320$ column (for $240 \times 320$ pixel images for PocketPCs); the picture is displayed on the PDA with the text, if any, on top.

The Answer column specifies the correct answer for each trial (or for the instructions, the key that should be pressed in order to advance to the next screen); the keys available for use are the scroll up and down keys, and the other four function keys; it is also possible to specify that the correct response is not to press any key. The coding FL, L, UP, DN, R, and FR was developed on the basis of the "classic" PDA key layout ${ }^{3}$ (which can be found, for instance, on Palm Tungsten E2s, T|Xs, and Treos, as well as on Dell Axims and most HP iPAQs), as is illustrated on the left in Figure 2.

The Duration column indicates how long the screen should be shown, and the Delay column specifies the intertrial interval - in other words, the blank space between the offset of the stimulus (or instructions screen) presented in the current trial and the presentation of the next stimulus (or instructions screen). If nothing is specified in the Delay column, the default is $250 \mathrm{msec}$. Responses (keypresses) made during the intertrial interval will be recorded; the timer starts running with the presentation of a given stimulus and does not stop until either a response is made or the next stimulus appears.

The MiniCog program automatically proceeds to the next screen after $1 \mathrm{~min}$, and if $2 \mathrm{~min}$ pass without input from the user, the program will terminate altogether, and any data will not be saved or scored. ${ }^{4}$ Because we want to give users the maximum time possible to read the instructions in our Stroop task example, these screens are set to display for $59.7 \mathrm{sec}$, with an intertrial interval of 


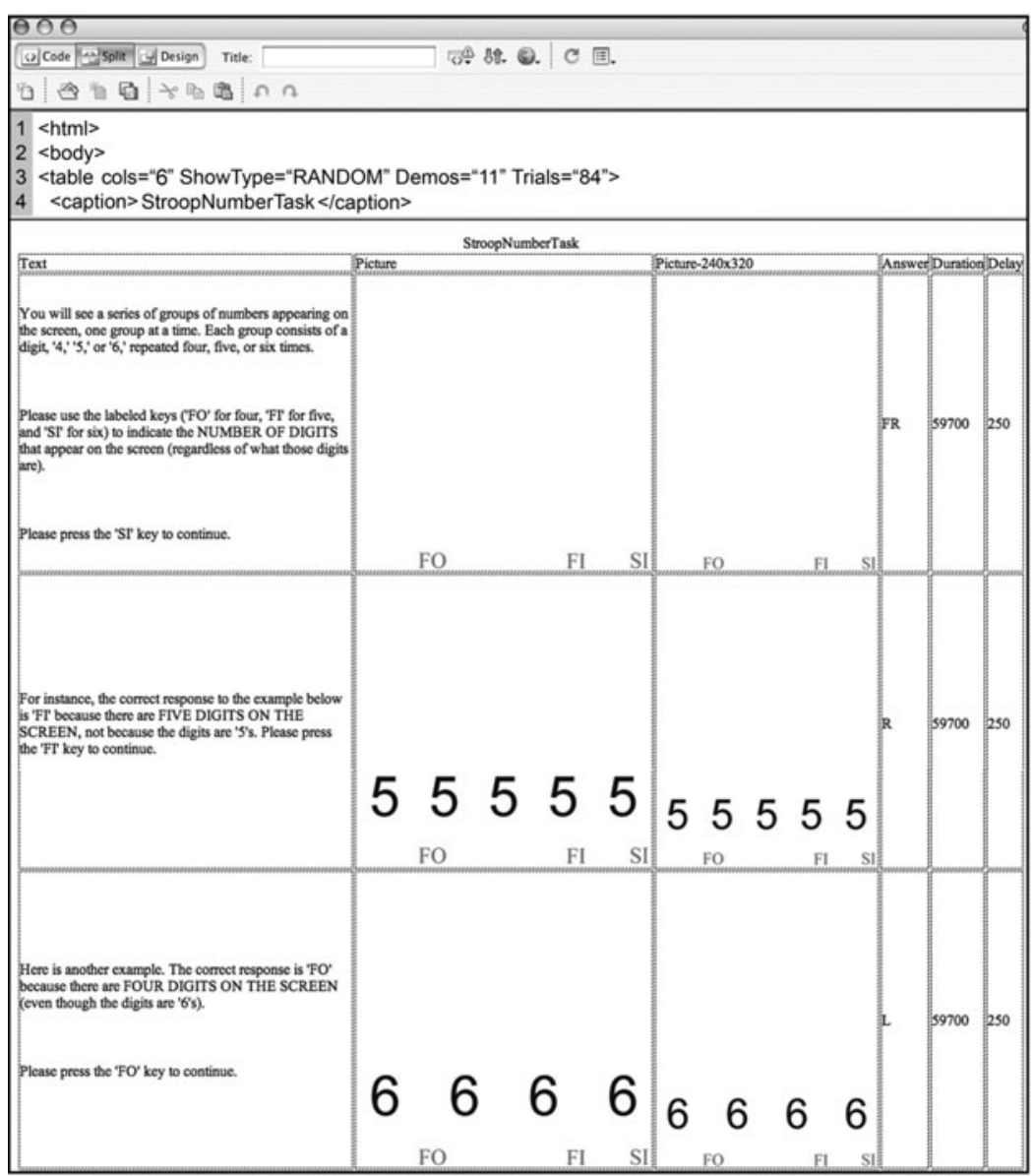

Figure 1. HTML table and coding for a typical task. A numeric Stroop task is illustrated in this example. The full HTML file for this task has 11 rows of demos (instructions and practice trials) and 12 rows of stimuli (the 12 stimuli are repeated approximately 7 times each, for a total of 84 trials).

$250 \mathrm{msec}$. (Note that if an investigator wants a picture to flash on the screen for a brief duration, followed by a long intertrial interval in which users can make their responses, those parameters can be set.)

\section{HTML Source Code}

In addition to the table, the HTML source code is used to script certain aspects of a task.

Classifying trials. In order to implement automatic scoring, MiniCog has to be able to distinguish between the rows in the table that correspond to instructions and practice and those that represent actual (test or scored) trials in the task; the Demos feature accomplishes this. Setting the Demos attribute (e.g., Demos $=$ " 11 " in the Stroop task example) indicates that the first $n$ rows (in this case, 11) in the table are instructions and/or practice trials and are not to be scored. During the demos (instructions and practice trials), the PDA will beep (if the sound is turned on) when a user presses the wrong key to proceed to the next screen. The PDA will not beep for incorrect answers once the test trials have begun.

The Trials attribute (e.g., Trials =" 84 " in the Stroop task example) tells the program how many nondemo trials (i.e., test trials) to present to the user and to score (in this case, 84).

A trial can consist of only one screen and, therefore, corresponds to a single row in the table. However, the total

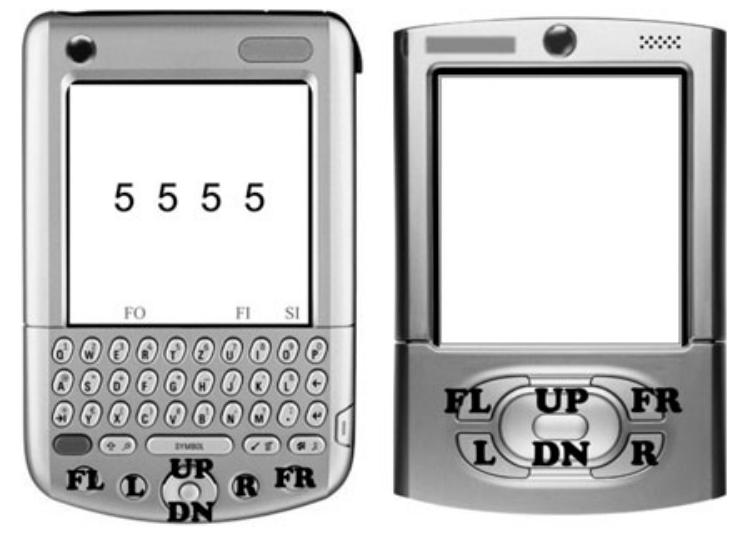

Figure 2. Classic key layout and labels (left). The figure on the right shows how the MiniCog coding maps onto another common key layout (used, for instance, on Palm Tungsten T3s). 
number of trials need not necessarily correspond to the number of nondemo rows in the table. Some tasks may have a limited number of stimuli (each occupying one row of the table) that are repeated. For instance, the Stroop task described here has 12 stimuli (an equal number of congruent and incongruent sequences of the digits 4, 5, and 6), occupying 12 rows, that are repeated approximately seven times each (depending on the randomization algorithm), for a total of 84 trials. Other tasks (those that may be administered repeatedly to the same user) may have a large pool of stimuli, only a subset of which are used in any given administration of the task.

Stimulus presentation order. By default, the trials are shown sequentially, and the table rows are assumed to be in sequential order. (The demos - instructions and practice trials - will always be sequential.) However, a fixed presentation order is not appropriate if the stimuli will be repeated, either within (as with our Stroop task example) or across administrations; it would be too easy for the users simply to memorize the answers.
The ShowType attribute (e.g., Showtype="Random" in the Stroop task example) is used to specify the order in which the stimuli will be presented. The investigator may specify a sequential order, a random order in which the program randomly selects each trial from among all the nondemo rows of the table (useful if a small number of stimuli are repeated), or a random-without-replacement order, where the program randomly selects each trial from among all the nondemo rows of the table but then removes that row from consideration (which is useful when the investigator wants each stimulus to be displayed just once). Moreover, the investigator can include more stimuli in the script (using more rows of the table) than will be presented in a given administration of the task. If the randomwithout-replacement option is used in this case, MiniCog will keep track of the stimuli shown in a given administration of the task to a given user and will remove those stimuli from the pool for the next administration to that user until all the stimuli have been shown once, at which point MiniCog begins again with the entire pool. This fea-

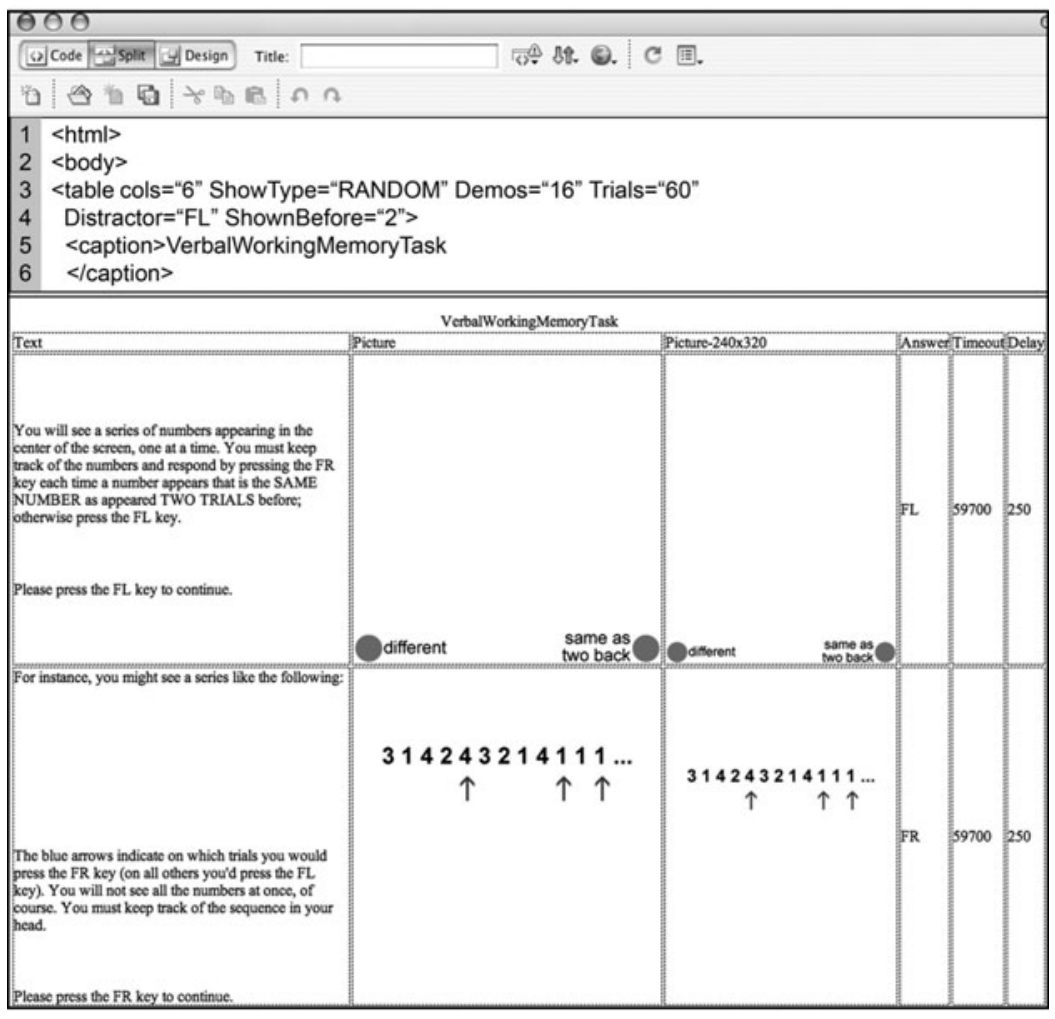

Figure 3. HTML table and coding for an $\boldsymbol{n}$-back task. The instructions and practice trials are implemented as with any other task; however, there is additional source code (ShownBefore $=$ "2," Distractor $=$ "FL") that indicates that this is a 2-back task and that the FL key is the correct response to the distractors (trials on which the displayed number is different from the one that had appeared two trials previously). Stimuli for such tasks must always be displayed in a random order (ShowType="Random"). In the table itself, the Answer cell for each test trial row (not shown) would contain "FR," since any stimulus could be a target (a trial on which the displayed number is the same as the one that had appeared two trials previously); MiniCog keeps track of the order of stimulus presentation when the task is administered, classifies each trial as a target (FR) or a distractor (FL), depending on the order, and scores the users' responses accordingly. 

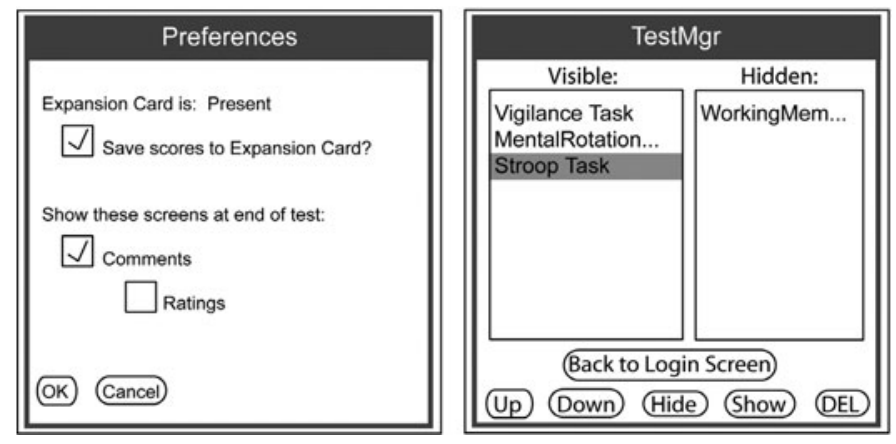

Figure 4. Preferences and Test Manager options.

ture is useful if users will be tested several times with the same task; for instance, if there are 80 rows of stimuli in the table, but only 20 trials in each administration of the task, users will see only a quarter of the stimuli each time, reducing the familiarity of any particular stimulus.

Additional features. We wanted to enable automatic scoring of a particular type of working memory task, designated an $n$-back task (see Figure 3), in which users must keep track of a series of stimuli and respond with a particular key when the stimulus they are currently seeing is the same as the one they saw $n$ back in the sequence (say, two trials prior; see Cohen et al., 1994). In order to score such tasks while still preserving the ability to randomize presentation of these stimuli, we created two special attributes, Distractor and ShownBefore. (These features are described in more detail in the user manual.)

\section{CONVERTING HTML SCRIPTS TO MINICOG TASKS USING MINICOG WRITER}

MiniCogWriter runs on any operating system featuring Java, including Mac OS, Unix, and Windows, and produces as output a palm resource code (PRC) or MiniCog Test (MCT) file that is compatible with MiniCog on the Palm or Windows Mobile platform, respectively.

\section{THE MINICOG APPLICATION}

The tool for administering the tasks is MiniCog; this application and the tasks must be installed on a PDA.

\section{Running MiniCog}

Upon running the program, the investigator can manage the list and order of tasks to be displayed (if multiple tasks are installed) and can set preferences specifying whether data should be saved to an expansion card and whether users will be asked to rate and comment on their own performance at the end of a task (see Figure 4).

Regardless of the specific task(s) loaded into the program, MiniCog displays a number of standard screens to all users (see Figure 5). It starts with a Login screen, which requires an ID (up to 10 characters) and permits the user to enter notes (up to 199 characters).
If the Login ID is new, a license agreement will appear, followed by a Demographics screen. The user must agree to the license terms in order to be able to use the software. The user must enter at least one character for each of the demographic items, but the program will accept meaningless data.

For a user to be considered the same (e.g., for purposes of saving to a specific datafile), the user must use the same Login ID each time. If the same Login ID is used more than once (for instance, if a given user is tested repeatedly on the same PDA), the license agreement and the Demographics screens are bypassed, and the user proceeds straight to the Task List.

After users complete a task, they may be asked to comment on or rate their performance (depending on the investigator's preference settings). If users are expected to complete more tasks, they may either proceed directly to the next task on the list or go back to select from the list of tasks. Users may also view their results (if in possession of the passcode; we do not recommend this for most studies) or log out of the program.

If the correct passcode is entered, users may view their current scores, both as summary text and as histograms (if other data exist with which to compare the users' current scores; see Figure 6).

\section{DATAFILES}

MiniCog saves two types of datafiles: USER files and SCORE files (e.g., A132Z-USER and StroopNumberTask$S C O R E) .{ }^{5}$ The SCORE files for each task provide the summary statistics for each user for each administration of that task, and the USER files give the detailed trial-bytrial data for a given user for each administration of each task (see Figure 7).

The SCORE file provides the Login ID and demographic input for all users who completed that task, as well as the time and date they performed the task, the number of items they answered correctly, their ER (percentage), the mean and $S D$ of their RTs for the correct trials, their throughput, and their ratings of their performance (if that preference was set by the investigator).

The USER files provide the trial-by-trial data - the trial indicator (i.e., row of the HTML table), correct answer, 


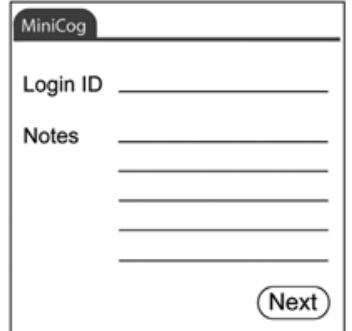

A

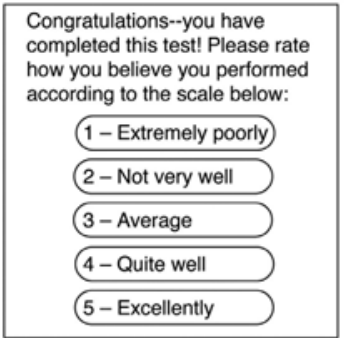

$\mathrm{D}$

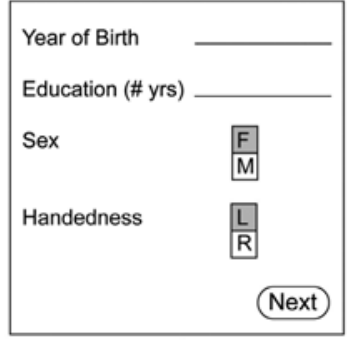

B

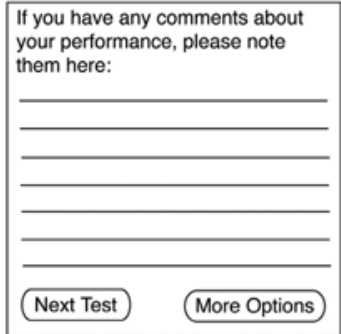

$\mathrm{E}$

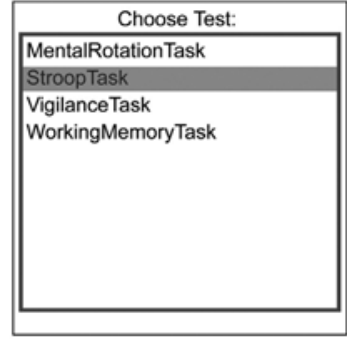

$\mathrm{C}$

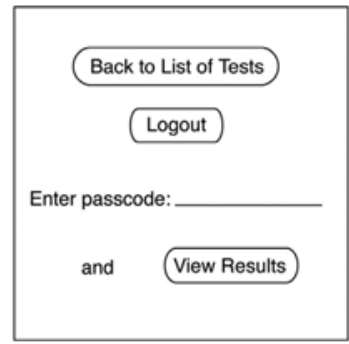

$\mathrm{F}$

Figure 5. MiniCog Login (A), Demographics (B), and Task List (C) screens. Users may be asked to rate their performance at the end of each task (D) and then to enter comments (E); if they select "Next Test" on the Comments screen, they will be taken to the next task in the list; if they select "More Options," they are taken to the Passcode screen (F).

time-and-date stamp, user response, and response timefor each administration of each task to a particular user (e.g., A132Z); these files also specify the session notes for each login and the demographic information entered at the time of the first login, as well as the users' ratings of, and comments about, their performance of each task (if that preference had been set by the investigator).

\section{Importing a Baseline Distribution}

MiniCog can display histograms showing an individual's performance relative to that of an aggregate population or to the user's own past performance. Because it is not very useful to display histograms with just a few scores, an investigator can create a file of aggregate scores, taken from different users tested on different PDAs. In other words,

\begin{tabular}{|ll||}
\hline \multicolumn{2}{|c|}{ Test Completed } \\
Number Correct & 35 \\
Error Rate (\%) & 8.75 \\
Mean RT (msecs) & 370 \\
Median RT & 367 \\
Std Dev RT & 181 \\
Max RT & 605 \\
Min RT & 184 \\
Throughput & 384 \\
\multicolumn{2}{|c|}{ Show Distribution Graphs } \\
Next Test \\
\hline
\end{tabular}

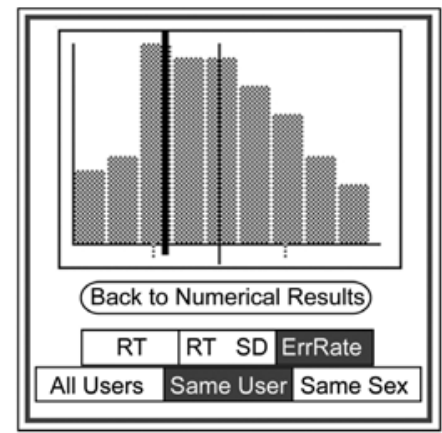

Figure 6. Results screens. The results (number correct; error rate [ER]; maximum, minimum, mean, and median response time [RT]; RT standard deviation; and throughput) are presented numerically (left). If there are enough data stored on the PDA, users can also view histograms displaying their current mean RT or ER (thick black line), compared with the means of all users or with the user's own past performance (right). The thin black line indicates the mean for the histogram; the two ticks on the $x$-axis indicate one standard deviation above and below the mean. (The bars are bins in the histogram function and may represent more than one user and/or more than one administration of the task.) 


\begin{tabular}{|c|c|c|c|c|c|c|c|c|c|c|c|}
\hline $\begin{array}{l}\text { StroopNumbe } \\
\text { Task-SCORE }\end{array}$ & & & & & & & & & & & \\
\hline LOGIN ID & \begin{tabular}{|c|} 
YEAR \\
OF \\
BIRTH
\end{tabular} & EDUCATION & SEX & $\begin{array}{c}\text { HANDED } \\
\text { NESS }\end{array}$ & $\begin{array}{c}\text { TIME } \\
\text { OF } \\
\text { TEST }\end{array}$ & $\begin{array}{c}\text { NUMBER } \\
\text { CORRECT }\end{array}$ & $\begin{array}{c}\text { AVERAGE } \\
\text { RESPONSE } \\
\text { TIME(MSECS) }\end{array}$ & \begin{tabular}{|c|} 
ERROR \\
RATE $(\%)$
\end{tabular} & $\begin{array}{l}\text { SD } \\
\text { (MS) }\end{array}$ & $\begin{array}{c}\text { TPUT } \\
\text { (/S) }\end{array}$ & RAT \\
\hline BB345 & 1967 & 17 & M & L & \begin{tabular}{|l|}
$3 / 23 / 05$ \\
$13: 04$ \\
\end{tabular} & 80 & 667 & 4.76 & 154 & 1.291 & 4 \\
\hline BB345 & 1967 & 17 & M & L & \begin{tabular}{|l|}
$3 / 24 / 05$ \\
$13: 25$ \\
\end{tabular} & 70 & 845 & 16.67 & 210 & .912 & 2 \\
\hline A132Z & 1971 & 12 & $F$ & R & \begin{tabular}{|l|}
$3 / 24 / 05$ \\
$16: 30$ \\
\end{tabular} & 82 & 702 & 2.38 & 123 & 1.243 & 3 \\
\hline
\end{tabular}

\begin{tabular}{|c|c|c|c|c|c|c|}
\hline A132Z-USER & & & & & & \\
\hline SESSION NOTES & TIMESTAMP & & & & & \\
\hline feeling tired today & $3 / 24 / 05$ & & & & & \\
\hline TEST NAME & TRIAL \# & TIMESTAMP & TRIAL INDEX & INPUT & $\begin{array}{c}\text { EXPECTED } \\
\text { ANSWER }\end{array}$ & $\begin{array}{l}\text { ELAPSED } \\
\text { TIME }\end{array}$ \\
\hline StroopNumberTask & 0 & $3 / 24 / 0516: 28$ & 9 & $\mathrm{~L}$ & $\mathrm{~L}$ & 659 \\
\hline StroopNumberTask & 1 & $3 / 24 / 0516: 28$ & 3 & $\mathrm{FR}$ & $\mathrm{R}$ & 581 \\
\hline StroopNumberTask & 2 & $3 / 24 / 0516: 28$ & 12 & $\mathrm{R}$ & $\mathrm{R}$ & 680 \\
\hline . & . & . & . & . & . & . \\
\hline . & . & . & . & . & . & . \\
\hline . & . & . & . & . & . & . \\
\hline StroopNumberTask & 6 & $3 / 24 / 0516: 30$ & 8 & FR & FR & 708 \\
\hline TEST NAME & RATING & \multicolumn{5}{|c|}{ COMMENT } \\
\hline StroopNumberTask & 3 & \multicolumn{5}{|c|}{ i felt a bit slow on this task but i think i was accurate } \\
\hline
\end{tabular}

Figure 7. Partial SCORE (top) and USER (bottom) datafiles (from an Excel spreadsheet); the ellipses in the USER file indicate that table rows have been deleted for this illustration.

a composite SCORE file can be created for each task by copying and pasting data from users of different PDAs into a single file that is then downloaded to one or more PDAs.

\section{DOCUMENTATION AND SPECIFICATIONS}

\section{System Requirements}

The current version ${ }^{6}$ of MiniCog is designed for Palm OS 5.2, Windows Mobile 2003, and later versions. It requires only about $100 \mathrm{~K}$ of memory; however, each task may require up to several megabytes of memory, depending on the number of picture files included. We recommend a PDA with at least $32 \mathrm{Mb}$ of memory. ${ }^{7}$ The PDA screen resolution must be at least $320 \times 320$ pixels (for Palms) and $240 \times 320$ pixels (for Pocket PCs); this is common among current PDA models.

MiniCogWriter will run on any platform (including Unix) that supports Java.

\section{Timing}

In principle, MiniCog should be accurate to $10 \mathrm{msec}$ on Palms (the Palm OS timer resolution) and at least as accurate on Pocket PCs. However, we have not conducted systematic testing of the timing.

\section{Limitations}

Some Palm units lose all installed software and accompanying data when the power is lost (for instance, if the battery dies after a long period of disuse or due to cold temperatures). Therefore, we recommend the use of a memory card; if one is present, data are saved to it automatically (unless the investigator changes this preference). The application itself can be stored on and launched from the memory card.

At the time of the software development, most PDAs had limited available memory (usually less than $64 \mathrm{Mb}$ ), and thus the software had to be relatively simple. MiniCog is not currently capable of presenting complex tasks in which each trial consists of multiple events (for instance, a fixation point followed by a series of pictures). Although such a trial design could be cobbled together, neither the automatic scoring nor the randomization features would work. It also cannot implement branching based on user responses. However, the program has been used to construct a number of simple cognitive tasks, including verbal and spatial $n$-back working memory tasks, Stroop tasks, a truelfalse verbal task, a sameldifferent mental rotation task, and others (for a description of these tasks, see Shephard \& Kosslyn, 2005).

Currently, the automatic scoring indicates correct versus incorrect responses but does not distinguish between different sorts of errors; for example, the program cannot calculate miss versus false alarm rates when users must make one response to target trials and a different response to distractors. (The investigators can make this calculation themselves, however, by manipulating the USER files directly once they have been uploaded to a desktop computer.)

Currently, MiniCog accepts single-keypress responses from only six buttons and, thus, cannot present tasks with multiple-choice responses requiring more than six choices 
or tasks requiring multiple-keypress responses (typed-in words, for instance). Although the keys on a PDA are small and closely spaced, we have observed that users do not have great difficulty with single-key responses. Text entry is slower than on a PC; however, not much text entry is required in order to use MiniCog.

This implementation does not support audio stimuli; the only auditory capability of the program is the feedback beep in response to incorrect user responses during the demos.

\section{CONCLUSION}

To our knowledge, MiniCog and MiniCogWriter are the first software package to give investigators the capability of implementing their own psychological tests and experiments for administration and scoring on a PDA. Such software may be a useful tool for investigators who wish to test users "in the field" or who require users to test themselves repeatedly on particular tasks.

We developed this software as part of our effort to create an extremely lightweight, portable, and inexpensive cognitive test battery for astronauts. Using MiniCog, we have already devised a number of quick psychological tests for cognitive assessment that are currently being validated in the laboratory and in the field (Shephard \& Kosslyn, 2005).

\section{Distribution and Documentation}

MiniCog and MiniCogWriter are not yet available to the general public. However, they are freely distributed to investigators conducting academic research and willing to sign a license agreement. More details can be found at minicog.wjh.harvard.edu.

A user manual is included with the software; this manual expands on the information included in this article and is kept up to date regarding modifications to the software. Bugs may be reported to minicog@wjh.harvard.edu, but the software is provided "as is," without warranties of any kind.

\section{REFERENCES}

Cohen, J. D., Forman, S. D., Braver, T. S., Casey, B. J., ServanSchreiber, D., \& Noll, D. C. (1994). Activation of the prefrontal cortex in a nonspatial working memory task with functional MRI. Human Brain Mapping, 1, 293-304.

Cohen, J. [D.], MacWhinney, B., Flatt, M., \& Provost, J. (1993). PsyScope: An interactive graphic system for designing and controlling experiments in the psychology laboratory using Macintosh computers. Behavior Research Methods, Instruments, \& Computers, 25, 257-271.

Lautzenhiser, A. L. (2007). Psychology and psychotherapy. Retrieved January 26, 2007 from www.access-company.com/support/ expertguides/psychology/index.html.

PDA resources selected by the University of Hong Kong Libraries (n.d.). Retrieved January 26, 2007 from sunzi1.lib.hku.hk/ER/format/hkul/ pda.

Shephard, J. M., \& Kosslyn, S. M. (2005). The MiniCog Rapid Assessment Battery: Developing a "blood pressure cuff for the mind." Aviation, Space, \& Environmental Medicine, 76, B192-B197.

Stroop, J. R. (1935). Studies of interference in serial verbal reactions. Journal of Experimental Psychology, 18, 643-662.

\section{NOTES}

1. NASA has approved the iPAQ h5550 for flight, and astronauts have them on board for "personal use."

2. Note that HTML was chosen because the text format is structured and there are a number of tools readily available for editing it; MiniCog is not a Web application, nor can the HTML scripts be run on a desktop computer.

3. MiniCog works on PDAs with other key layouts, but investigators may have to discover the key coding by trial and error.

4. Many PDAs shut off automatically after a maximum of $3 \mathrm{~min}$ without input, in any case.

5. On Palms, these are saved as PDB (palm database) files, which can be converted into CSV (comma separated value) text files and opened in a standard spreadsheet program (e.g., Microsoft Excel). We distribute a program called $p d b 2 \operatorname{csv}$ (and there are other programs available for download over the Web) for converting the files (see minicog.wjh harvard.edu).

6. We plan a final release of this software in spring 2007 , and this may include some features in addition to those described here.

7. The test battery (MRAB) that Shephard and Kosslyn (2005) have developed needs more than $32 \mathrm{Mb}$ of memory, in addition to a specific key layout, and so requires a Palm Tungsten $\mathrm{C}$ for administration. However, there are a number of PDAs that meet the general specifications for MiniCog; for instance, see www.palmsource.com/products/, www.dell .com/axim, or www.hp.com/country/us/en/prodserv/handheld.html.
(Manuscript received March 10, 2005; revision accepted for publication August 31, 2005.) 